\title{
25 Research Square \\ Infective patterns of cryptococcosis in patients with connective tissue disease:a retrospective study
}

\section{Minxi Lao}

Sun Yat-sen University First Affiliated Hospital

Yingying Gong ( $\nabla$ gongyy01@126.com )

the first affiliated hospital of Sun yat-sen university

\section{Chuyu Shen}

Sun Yat-sen University First Affiliated Hospital

\section{Cuicui Wang}

Sun Yat-sen University First Affiliated Hospital

\section{Liuqin Liang}

Sun Yat-sen University First Affiliated Hospital

\section{Zhongping Zhan}

Sun Yat-sen University First Affiliated Hospital

\section{Dongying Chen}

Sun Yat-sen University First Affiliated Hospital

\section{Research article}

Keywords: connective tissue disease, cryptococcosis, glucocorticoid

Posted Date: July 18th, 2019

DOI: https://doi.org/10.21203/rs.2.11666/v1

License: (c) (1) This work is licensed under a Creative Commons Attribution 4.0 International License.

Read Full License

Version of Record: A version of this preprint was published at Clinical Rheumatology on April 3rd, 2020. See the published version at https://doi.org/10.1007/s10067-020-05068-6. 


\section{Abstract}

Background: Cryptococcal infection is a life-threatening complication in patients with connective tissue disease (CTD). This retrospective study was performed to explore the clinical features and associated factors of cryptococcosis in patients with CTD from southern China. Methods: Demographic and clinical data were collected between 2007 and 2018. Associated factors were analyzed by logistic regression analysis. Results: A total of 6809 inpatients with CTD were included. Cryptococcosis was diagnosed in 30 patients (prevalence, $0.4 \%$ ). Cryptococcosis was predominant in patients with ANCA-associated vasculitis (AAV) (prevalence, 6/530, 1.1\%). Lung was commonly involved (18/30,60.0\%), followed by meninges $(6 / 30,20.0 \%)$, blood stream $(5 / 30,16.7 \%)$ and disseminated cryptococcosis $(1 / 30,3.3 \%)$. Infiltrates $(10 / 18,55.6 \%)$ and small nodules $(8 / 18,44.4 \%)$ were the main radiographic manifestation of pulmonary cryptococcosis $(\mathrm{PC})$. The positive rate of serum cryptococcal antigen ( $\mathrm{CrAg})$ in patients with PC was $88.2 \%$. Cryptococcus spp. were found in $75 \%(3 / 4)$ patients who underwent lung biopsy. Most of the patients with cryptococcal meninges (CM) had elevated cerebrospinal fluid (CSF) opening pressure (6/7, $85.7 \%)$ and decreased CSF glucose level $(5 / 7,71.4 \%)$. Positive blood culture confirmed the diagnosis of cryptococcal sepsis (CS). Three patients died (10.0\%), including one with CM and two with PC.

Multivariate logistic regression analysis showed that accumulated dose of glucocorticoid (GC) [odds ratio $(O R)=1.42,95 \%$ confidence interval $(\mathrm{Cl}) 1.08-1.87, \mathrm{P}=0.01]$ was associated with cryptococcosis in patients with CTD. Conclusions: Cryptococcosis developed in various organs. Typical radiological manifestation accompanied with positive serum CrAg were helpful clues for diagnosis. Lumbar puncture was a critical diagnostic method to distinguish $\mathrm{CM}$. The accumulated dose of GC was associated with cryptococcosis in patients with CTD.

\section{Background}

Infection is one of the major threats to patients with connective tissue disease (CTD) because of the immune disturbance and immunosuppressive therapy [1,2]. Cryptococcosis was a common occurring invasive fungal disease (IFD) in immunocompromised hosts such as patients with acquired immune deficiency syndrome (AIDS) and organ transplantation [3]. Patients with cryptococcosis could be asymptomatic or develop organ failure based on the infected species and host immunity. Until recently, research on cryptococcosis mainly focused on the AIDS population. The infective profile in patients with CTD remained to be elucidated. The incidence rate of cryptococcosis in patients with systemic lupus erythematosus (SLE) was nearly $0.5 \%$, mainly distributed in Asian countries ( $67.3 \%)$ [4]. Approximately $0.2 \%$ of the patients with rheumatoid arthritis (RA) complicated with cryptococcosis [5]. Cryptococcosis in other CTD such as systemic vasculitis was only reported in scattered cases [6, 7]. Asia is the endemic region for cryptococcosis,[8] and the incidence and features of infection differed geographically. Thus, to explore the situation of cryptococcosis in CTD in China could provide useful information for the management of such a comorbidity $[9,10]$.

In this study, we conducted a retrospective analysis, aiming to identify the epidemiology, clinical profile and risk factors of cryptococcosis in patients with CTD from southern China. 


\section{Methods}

\section{Study design}

We performed a retrospective study on the inpatients from the First Affiliated Hospital of Sun Yat-Sen University from January $1^{\text {st }}, 2007$ to December $31^{\text {st }}, 2018$. The ICD-10 coding of discharged diagnoses was used to identify patients with CTD and cryptococcosis (relevant ICD-10 coding in Appendix 1). SLE was established using the 1997 American College of Rheumatology classification criteria [11]. ANCAassociated vasculitis (AAV) was determined according to the definitions of the Chapel Hill nomenclature $[12,13]$. RA was diagnosed based on the 1987 ACR revised criteria for the classification of rheumatoid arthritis [14]. Inflammatory myopathy (IM) was established referring to the classification criteria proposed by Bohan [15]. The diagnosis of systemic sclerosis (SSc) was conducted based on 1980 ARA classification criteria for SSc [16]. Primary Sjogren syndrome (SS) was defined according to the American-European Consensus Group classification criteria for SS [17]. Mixed connective tissue disease (MCTD) was considered based on the Sharp criteria [18]. Ethics committee of the First Affiliated Hospital of Sun Yat-sen University approved the research. This work was conducted in compliance with the Declaration of Helsinki principles.

\section{Case definition}

Cryptococcosis was determined using the EORTC/MSG 2008 Guidelines [19] and classified into three categories as proven, probable, and possible. In the interest of capturing cases which were most likely to be truly cryptococcosis, we only included proven and probable cases in this research.

Pulmonary cryptococcosis (PC) was diagnosed on the basic of i) presence of one of the computed tomography (CT) signs (dense, well-circumscribed lesions with or without a halo sign, or air-crescent sign, or cavity); ii) Cryptococcus spp. in sputum, bronchoalveolar lavage fluid (BALF) or serum cryptococcal antigen ( $\mathrm{CrAg}$ ) positive; iii) histopathologic examination of lung tissue finding Cryptococcus spp. or recovery of Cryptococcus spp. in pleural fluid culture. Patients with CTD having criteria (iii) were considered as proven cases. Patients with CTD satisfying criteria (i) and (ii) were considered as probable cases. Patients presenting criteria (i) only were classified as possible cases. Cryptococcal meningitis (CM) was diagnosed as proven if Cryptococcus spp. were found in cerebrospinal fluid (CSF). Probable case was considered if the patients with CTD presented meningeal enhancement on cranial magnetic resonance imaging (MRI) or CT and positive CrAg test in serum or CSF sample. Patients with cranial radiographic manifestation only were considered as possible CM. Cryptococcal sepsis (CS) was established if blood culture yields Cryptococcus spp. Patients with incomplete data were excluded.

Inpatients with CTD but no history of any infection from the same period of time were chosen as controls. Four controls per case were randomly selected among all potential controls matching with the cases by age, gender, subtype, and the duration of CTD (from CTD onset to cryptococcosis).

\section{Outcome}


All the patients were followed until discharge. Treatment outcome was classified into two categories including improvement and progression. Improvement was defined as clinical symptoms and radiographic imaging partially resolved. Progressive disease was defined as clinical symptoms and radiographic imaging worsened.

\section{Clinical variables}

Demographic and clinical data were collected from medical records. Clinical characteristics of cryptococcosis included symptoms and signs, sites of infection and treatment regimen. The degree of fever was classified as mild $\left(37.1-38^{\circ} \mathrm{C}\right)$, moderate $\left(38.1-39^{\circ} \mathrm{C}\right)$ and high $\left(>39^{\circ} \mathrm{C}\right)$. Cryptococcal capsule polyglycan antigen was detected by latex agglutination (LA) test. Specimen culture and biopsy finding were recorded if available. Medication history included daily dose of glucocorticoid (GC) and the use of immunosuppressive agents within the three months prior to cryptococcosis.

\section{Statistical analysis}

The descriptive variables included mean (standard deviation, SD) or median (interquartile range, IQR) depending on the distribution of continuous variables and number (percentage) for qualitative variables. Between-group comparison was evaluated with chi-square test or Fisher exact test for categorical variables and Student's $t$-test for continuous variables with normal distribution. Between-group comparison was evaluated using the Mann-Whitney $U$ test for continuous variables with non-normal distribution. Variables with clinical significance and $P$ value $<0.10$ from the univariate analysis were considered as candidates for the multivariate analysis. The multivariate analysis was preceded by a forward procedure to identify the factors associated with cryptococcosis. Associated factors were reported with $P$ values, odds ratio (OR), and $95 \%$ confidence interval $(\mathrm{Cl})$. The level of significance was set at $P<0.05$. All statistical analysis was performed with the SPSS 19.0 statistical package (SPSS Inc, Chicago, IL, USA).

\section{Results}

\section{Basic characteristics}

A total of 6809 inpatients with CTD (including 4196 patients with SLE, 728 with IM, 666 with primary SS, 530 with AAV, 407 with RA, 197 with SSc, and 85 with MCTD) were enrolled. Cryptococcosis occurred in 30 patients. Proven cases were diagnosed in 14 (46.7\%) patients, and probable in 16 (53.3\%) patients. The hospital-based prevalence of cryptococcosis in patients with CTD was $0.4 \%$ (30/6809). Patients with AAV had the highest prevalence rate $(6 / 530,1.1 \%)$, followed by RA $(4 / 407,1.0 \%)$, and SLE $(20 / 4196$, $0.5 \%$ ). Mean (SD) age was $45.5 \pm 16.3$ years (range, 19-78). Male-to-female ratio was $8: 22$. The median (IQR) duration of CTD was 24.0 months $(6.0,78.0)$. Four (13.3\%) patients were dialysis-dependent. No patients were infected by HIV or received organ transplantation. Exposure to pigeon droppings, or keeping cats, dogs or poultry was not reported. Characteristics of patients with CTD and cryptococcosis were shown in Table 1. 
During the three months prior to cryptococcosis, 73.3\% (22/30) patients were treated with GC, and 1 (3.3\%) patient received intravenous methylprednisolone pulse therapy. Immunosuppressants were prescribed to 18 (60.0\%) patients. In particular, mycophenolate mofetil (MMF), cyclophosphamide (CYC), methotrexate (MTX), cyclosporin A (CsA), and azathioprine (AZA) were given to 7 (23.3\%), 6 (20.0\%), 2 (6.7\%), 2 (6.7\%), and 1 (3.3\%) patients, respectively.

\section{Pulmonary cryptococcosis}

PC was diagnosed in $18(60.0 \%)$ patients, including 8 patients with SLE, 6 with AAV, and 4 with RA. The average (SD) age was $51.1 \pm 17.8$ years. Male-to-female ratio was $8: 10$. The median (IQR) duration of CTD was $12.0(5.0,84.0)$ months. The median (IQR) time from symptoms onset to diagnosis was $17(5.5$, 46.0) days. Clinical symptoms included cough/sputum (38.9\%), mild-to-moderate fever (27.8\%), and dyspnea (11.1\%). No patients presented hemoptysis. Six (33.3\%) patients were asymptomatic and pulmonary diseases were detected by incidental radiological examination. Radiographic features of pulmonary cryptococcosis were diverse and summarized in Table 2 . Infiltrates (10/18, 55.6\%) and small nodules with diameter $<3 \mathrm{~cm}(8 / 18,44.4 \%)$ were the main manifestation (Figure $1 \mathrm{~A})$. Cavity was rare $(1 / 18,5.6 \%)$ (Figure 1B). Half of the nodules located adjacent to pleura. Five $(5 / 8,62.5 \%)$ cases presented single nodules while the rest $(3 / 8,37.5 \%)$ had multiple nodules. Most of the nodules displayed blurred edge (Figure 1C). The proportion of single lobe involvement (10/18,55.6\%) and multiple lobes involvement $(8 / 18,44.4 \%)$ was comparable. Three lobulated nodules were misdiagnosed with lung cancer (Figure 1D). The positivity was high in serum CrAg in patients with PC $(15 / 17,88.2 \%)$. The positive rate of sputum culture $(2 / 11,18.2 \%)$ and BALF culture $(1 / 8,12.5 \%)$ was relatively low. Lung biopsy was conducted in four patients showing pulmonary nodules (diameter $\geq 2 \mathrm{~cm}$ ) or consolidation, and encapsulated budding yeasts suggesting Cryptococcus spp. were found in three patients $(3 / 4,75.0 \%)$. Positive alcian blue staining $(3 / 4,75.0 \%)$ and periodic acid schiff (PAS) staining $(3 / 4,75.0 \%)$ further supported the diagnosis.

\section{Cryptococcal meningitis}

Seven $(23.30 \%)$ female patients with SLE were complicated with CM, and one of them was complicated with CS simultaneously. The average (SD) age was $40.6 \pm 7.7$ years. The median (IQR) duration of CTD was $72.0(48.0,120.0)$ months. The median (IQR) time from symptoms onset to diagnosis was 36.0 $(22.5,49.5)$ days. Headache was the most common symptoms $(85.7 \%)$, followed by mild fever $(57.1 \%)$, vomit (57.1\%), tinnitus (28.6\%) and diplopia (14.3\%). Serum CrAg was positive in five out of seven patients (71.4\%). Brain magnetic resonance imaging (MRI) was performed in two patients. Radiographic manifestations included spotted lesion scattered in cerebral cortex with hyperintensity in T2WI and a cyst near the pineal gland. All the patients received lumbar puncture. Characteristics of CSF were summarized in Table 3. Leukocytes were mildly elevated in all patients $(7 / 7,100 \%)$, and mononuclear cells were the major component $(78.4 \pm 10.4 \%)$. Glucose level decreased $(5 / 7,71.4 \%)$. CSF opening pressure was high in $85.7 \%(6 / 7)$ of the patients, and extremely elevated in the four of them $(66.7 \%)\left(>330 \mathrm{mmH}_{2} \mathrm{O}\right)$. 
Cryptococcus neoformans in CSF smear sample was identified by India ink in six (85.7\%) patients, and recovered by CSF culture in one (14.3\%) patient.

\section{Cryptococcal sepsis}

CS occurred in six (20.0\%) female lupus patients, and one of them also developed CM. The average (SD) age was $32.3 \pm 8.9$ years. The median (IQR) duration of CTD was $6.0(6.0,24.0)$ months. The median (IQR) time from symptoms onset to diagnosis was $6.0(5.0,7.0)$ days. Most of the patients $(5 / 6,83.3 \%)$ had high fever. Prior to CS, one patient had skin ulcer, and another one developed continuous ambulatory peritoneal dialysis (CAPD)-related peritonitis. Diagnosis was confirmed with positive blood culture obtained when the patients presented high fever. The positivity of serum CrAg was $40.0 \%(2 / 5)$.

\section{Treatment and prognosis}

The median (IQR) follow-up was $15.0(10.0,24.0)$ days. Intravenous fluconazole $(600 \mathrm{mg} / \mathrm{d})$ was given to 7 patients with $\mathrm{PC}$, and intravenous voriconazole $(400 \mathrm{mg}$ twice a day on day 1 , and $200 \mathrm{mg}$ twice a day from day 2) to 2 patients. Oral fluconazole was given to 5 patients with reduced dose of $300 \mathrm{mg} / \mathrm{d}$ due to impaired kidney function [creatinine clearance rate (Ccr) ranged from $17.3-29.8 \mathrm{ml} / \mathrm{min}$ ]. Three patients were treated with intravenous fluconazole $(600 \mathrm{mg} / \mathrm{d})$ after hemodialysis. One patient with PC received surgery and subsequent oral fluconazole $(200 \mathrm{mg} / \mathrm{d})$.

Five patients having $\mathrm{CM}$ received combined therapy with intravenous amphotericin $\mathrm{B}$ (started from $5 \mathrm{mg} / \mathrm{d}$, and escalated by $5 \mathrm{mg}$ per day until $0.5-0.7 \mathrm{mg} / \mathrm{kg} / \mathrm{d})$ and flucytosine $(100 \mathrm{mg} / \mathrm{kg} / \mathrm{d})$. One patient with $\mathrm{CM}$ was treated with oral fluconazole $(400 \mathrm{mg} / \mathrm{d})$ due to decreased $\mathrm{Ccr}(25.0 \mathrm{ml} / \mathrm{min})$.

Two patients with CS and one patient with $\mathrm{CM}$ and CS were treated with amphotericin B and flucytosine. Treatment algorithm was similar to that of $\mathrm{CM}$. Two patients with CS were given amphotericin $\mathrm{B}$ alone due to thrombocytopenia prior to antifungal therapy. One patient with CS was treated with oral fluconazole $(400 \mathrm{mg} / \mathrm{d})$ due to decreased $\operatorname{Ccr}(12.6 \mathrm{ml} / \mathrm{min})$.

The mortality rate in CTD patients with cryptococcosis was $10.0 \%(3 / 30)$. The underlying diseases were SLE $(n=1), \operatorname{RA}(n=1)$, and AAV $(n=1)$. The mortality rate of PC and PM was $11.1 \%(2 / 18)$ and $16.7 \%$ $(1 / 6)$, respectively. No patient with PS died. The duration from disease onset to diagnosis was 30,60 and 7 days in three patients, respectively. All the deceased patients ( 2 females, 1 male) died of co-infection with fungi and bacteria.

\section{Comparison between CTD patients with and without cryptococcosis}

A comparison between CTD patients with and without cryptococcosis was shown in Table 4. The serum IgG level was decreased in patients with cryptococcosis (11.0 vs $13.3 \mathrm{~g} / \mathrm{I} \otimes P=0.001)$, while CRP level increased $(23.3$ vs $2.5 \mathrm{mg} / \mathbb{I} \otimes P<0.001)$. The accumulated dose of $\mathrm{GC}$ within the three months before cryptococcosis onset was significantly higher in patients in infective group than those in the control 
group ( 0.9 vs $0.2 \mathrm{~g}, P=0.01$ ). Multivariate regression analysis showed that accumulated dose of $\mathrm{GC}$ increased the risk of cryptococcosis (OR 1.42, 95\% Cl 1.08-1.87, $P=0.01$ ) (Table 5).

\section{Discussion}

In the current research, we analyzed the infectious profile of cryptococcosis in patients with CTD from southern China. Cryptococcosis was a common complication in patients with CTD. The diagnosis of PC relied on pulmonary CT scan, serum CrAg detection and lung biopsy if possible. Lumbar puncture and blood culture were reliable methods to diagnose CM and CS, respectively.

Cryptococcosis could develop in various organs. In the current study, lung was the most frequently involved. Hemoptysis, which was common in pulmonary aspergillosis and lung cancer, was rare in cryptococcosis. Nodules, especially small nodules adjacent to the pleura were common radiographic manifestation [20], while cavitary lesions were less frequent compared to that in pulmonary aspergillosis [21]. Serum CrAg was a useful predictor for cryptococcal infection [22], and PAS-staining was a productive method to distinguish Cryptococcus spp. Generally, the diagnosis of PC depends on the assessment of clinical symptoms, radiographic manifestation, and etiological identification. PC should be taken into consideration in patients having pulmonary infiltrates or small nodules but inadequate response to antibiotics. Serum CrAg provided valuable clues for the diagnosis of cryptococcosis. Lung biopsy was suggested if diagnosis was still not confirmed through the aforementioned procedures, and PAS-staining was useful to identify the pathogens.

$\mathrm{CM}$ was a major pattern of cryptococcal infection in our study. Among three subtypes of cryptococcosis, both the duration of CTD and the diagnostic time was the longest in patients with CM. Slow onset of the disease with non-specific symptoms usually delayed the diagnosis. As reported, $38.2 \%$ of $\mathrm{CM}$ in lupus patients was misdiagnosed as non-fungal infection or active disease [4]. Diagnosis of CM was predominantly depended on lumbar puncture. Remarkably elevated CSF opening pressure with decreased glucose level implied CM. Diagnosis was confirmed by the isolation of Cryptococcus spp. in CSF by Indian ink or culture. Negativity once in CSF smear did not preclude CM, and repeated lumbar puncture was needed in suspected patients.

CS was not rare in patients with CTD and accounted for $20.0 \%$ of the cases in the current research, the prevalence compared to $11.1-19.6 \%$ in previous reports $[8,23]$. The characteristics of CS were less discussed. Of note, two patients had skin infection or conduit communicating with the outer space. Such patients were risky for CS once exposed to the contaminated air. Isolation of Cryptococcus spp. in blood was the unique method to diagnose CS. Therefore, blood culture in the case of high fever was necessary especially in patients with skin lesions.

Compared to aspergillosis which had a mortality rate of $31.3 \%$, the prognosis of cryptococcosis was relatively favorable [24]. Most of the infected patients improved after effective treatment $[25,26]$. A Chinese study compared the diagnostic time between CNS infection caused by fungi and bacteria, and found that it usually took longer time (average 16.8 days) in diagnosing fungal CNS infection than the 
non-tuberculosis bacteria groups (average 4.8 days), partially due to insidious or atypical clinical manifestations [23]. In the current study, two deceased patients experienced long period from disease onset to diagnosis, resulting in delayed treatment. Thus, increasing awareness of cryptococcosis might benefit the prognosis in patients with CTD.

GC was a risk factor for cryptococcosis in lupus patients $[23,26]$. Taking prednisone equivalent of $>30 \mathrm{mg} /$ day before infection increased the mortality in lupus patients with $\mathrm{CM}$ [4]. Our results further supported the previous finding and showed that accumulated dose of GC was associated with the development of cryptococcosis in patients with CTD. Other risk factors such as lymphopenia, hypoalbuminemia which were associated with aspergillosis[27] seemed to be less relevant in cryptococcosis.

Our research had certain limitations. The prevalence of cryptococcosis in patients with CTD could be underestimated since only inpatients were included. Clinical experience originating from one center limited its generalizability to other population. Besides, the cross-sectional nature had limited value in studying treatment response and long-term prognosis.

\section{Conclusions}

Cryptococcosis was a common opportunistic infection in patients with CTD. It could develop in various organs including lung, brain and blood stream. Typical manifestation accompanied with positive serum CrAg suggested PC. CM usually presented slow disease onset. Lumbar puncture was a critical diagnostic method. CS was not rare and blood culture was reliable and necessary in suspected patients. The accumulated dose of GC was associated with cryptococcosis in patients with CTD.

\section{Abbreviations}

AAV: ANCA-associated vasculitis; AIDS: acquired immune deficiency syndrome; AZA: azathioprine; BALF: bronchoalveolar lavage fluid; CrAg: cryptococcal antigen; Ccr: creatinine clearance rate; CM: cryptococcal meningitis; CRP: C-reactive protein; CS: cryptococcal sepsis; CsA: cyclosporin A; CSF: cerebrospinal fluid; CT: computed tomography; CTD: connective tissue disease; CYC: cyclophosphamide; ESR: erythrocyte sedimentation rate; GC: glucocorticoid; IFD: invasive fungal disease; IM: inflammatory myopathy; IQR: interquartile range; LA: latex agglutination; MCTD: mixed connective tissue disease; MMF: mycophenolate mofetil; MRI: magnetic resonance imaging; MTX: methotrexate; OR: odds ratio; PC: pulmonary cryptococcosis; PAS: periodic acid schiff; RA: rheumatoid arthritis; SD: standard deviation; SLE: systemic lupus erythematosus; SS: Sjogren syndrome; SSc: systemic sclerosis

\section{Declarations}

Acknowledgments: 


\section{Author contributions}

Dongying Chen and Zhongping Zhan contributed to study design and critical revision of the manuscript. Minxi Lao and Yingying Gong participated in data processing and were the major contributors in manuscript writing. Chuyu Shen participated in data analysis and interpretation. Cuicui Wang and Liuqin Liang assisted in data collection.

\section{Funding}

This project was supported by grants from the National Natural Science Foundation of China (81601403, 81603435), grants from the Natural Science Foundation of Guangdong Province (2018A0303130294) and grants from Guangdong Technology Project (2017A020215148).

\section{Availability of data and materials}

All data generated or analysed during this study are included in this published article.

\section{Ethics approval and consent to participate}

The research was ethically approved by Ethics committee of the First Affiliated Hospital of Sun Yat-sen University. This work was conducted in compliance with the Declaration of Helsinki principles. Patients' data were anonymized and kept confidential throughout this study.

\section{Consent for publication}

Not applicable.

\section{Competing interests}

None of the authors have any conflicts to declare. 


\section{Author details}

${ }^{1}$ Department of Rheumatology, The First Affiliated Hospital of Sun Yat-sen University, Guangzhou, China.

${ }^{2}$ Department of Geriatrics, The First Affiliated Hospital of Sun Yat-sen University, Guangzhou, China

\section{References}

1. Goldblatt F, Chambers S, Rahman A, Isenberg DA: Serious infections in British patients with systemic lupus erythematosus: hospitalisations and mortality. Lupus 2009, 18(8):682-689.

2. Yang L, Xie H, Liu Z, Chen Y, Wang J, Zhang H, Ge Y, Hu W: Risk factors for infectious complications of ANCA-associated vasculitis: a cohort study. BMC nephrology 2018, 19(1):138.

3. Maziarz EK, Perfect JR: Cryptococcosis. Infectious disease clinics of North America 2016, 30(1):179206.

4. Fang W, Chen M, Liu J, Hagen F, Ms A, Al H, Zhang P, Guo Y, Boekhout T, Deng D et al: Cryptococcal meningitis in systemic lupus erythematosus patients: pooled analysis and systematic review. Emerging microbes \& infections 2016, 5(9):e95.

5. Liao TL, Chen YM, Chen DY: Risk factors for cryptococcal infection among patients with rheumatoid arthritis receiving different immunosuppressive medications. Clinical microbiology and infection : the official publication of the European Society of Clinical Microbiology and Infectious Diseases 2016, 22(9):815.e811-815.e813.

6. Buchta V, Prasil P, Vejsova M, Mottl R, Kutova R, Drahosova M, Plisek S: Cryptococcus neoformans meningoencephalitis in a patient with polyarteritis nodosa. Folia microbiologica 2014, 59(6):515521.

7. Iwazu K, Iwazu Y, Takeda S, Akimoto T, Yumura W, Takahashi H, Ito C, Kanai K, Taniguchi N, Hirai Y et al: Successful treatment of serial opportunistic infections including disseminated nocardiosis and cryptococcal meningitis in a patient with ANCA-associated vasculitis. Internal medicine (Tokyo, Japan) 2012, 51(21):3051-3056.

8. Wang LR, Barber CE, Johnson AS, Barnabe C: Invasive fungal disease in systemic lupus erythematosus: a systematic review of disease characteristics, risk factors, and prognosis. Seminars in arthritis and rheumatism 2014, 44(3):325-330.

9. Galli M, Antinori S, Atzeni F, Meroni L, Riva A, Scire C, Adorni F, Quartuccio L, Sebastiani M, Airo P et al: Recommendations for the management of pulmonary fungal infections in patients with rheumatoid arthritis. Clinical and experimental rheumatology 2017, 35(6):1018-1028.

10. Yanagawa N, Sakai F, Takemura T, Ishikawa S, Takaki Y, Hishima T, Kamata N: Pulmonary cryptococcosis in rheumatoid arthritis (RA) patients: comparison of imaging characteristics among RA, acquired immunodeficiency syndrome, and immunocompetent patients. European journal of radiology 2013, 82(11):2035-2042.

11. Hochberg MC: Updating the American College of Rheumatology revised criteria for the classification of systemic lupus erythematosus. Arthritis and rheumatism 1997, 40(9):1725. 
12. Jennette JC, Falk RJ, Andrassy K, Bacon PA, Churg J, Gross WL, Hagen EC, Hoffman GS, Hunder GG, Kallenberg CG et al: Nomenclature of systemic vasculitides. Proposal of an international consensus conference. Arthritis and rheumatism 1994, 37(2):187-192.

13. Jennette JC: Overview of the 2012 revised International Chapel Hill Consensus Conference nomenclature of vasculitides. Clinical and experimental nephrology 2013, 17(5):603-606.

14. Arnett FC, Edworthy SM, Bloch DA, McShane DJ, Fries JF, Cooper NS, Healey LA, Kaplan SR, Liang $\mathrm{MH}$, Luthra HS et al: The American Rheumatism Association 1987 revised criteria for the classification of rheumatoid arthritis. Arthritis and rheumatism 1988, 31(3):315-324.

15. Bohan A, Peter JB: Polymyositis and dermatomyositis (first of two parts). The New England journal of medicine 1975, 292(7):344-347.

16. Preliminary criteria for the classification of systemic sclerosis (scleroderma). Subcommittee for scleroderma criteria of the American Rheumatism Association Diagnostic and Therapeutic Criteria Committee. Arthritis and rheumatism 1980, 23(5):581-590.

17. Vitali C, Bombardieri S, Jonsson R, Moutsopoulos HM, Alexander EL, Carsons SE, Daniels TE, Fox PC, Fox RI, Kassan SS et al: Classification criteria for Sjogren's syndrome: a revised version of the European criteria proposed by the American-European Consensus Group. Annals of the rheumatic diseases 2002, 61(6):554-558.

18. Sharp GC, Irvin WS, Tan EM, Gould RG, Holman HR: Mixed connective tissue disease-an apparently distinct rheumatic disease syndrome associated with a specific antibody to an extractable nuclear antigen (ENA). The American journal of medicine 1972, 52(2):148-159.

19. De Pauw B, Walsh TJ, Donnelly JP, Stevens DA, Edwards JE, Calandra T, Pappas PG, Maertens J, Lortholary O, Kauffman CA et al: Revised definitions of invasive fungal disease from the European Organization for Research and Treatment of Cancer/Invasive Fungal Infections Cooperative Group and the National Institute of Allergy and Infectious Diseases Mycoses Study Group (EORTC/MSG) Consensus Group. Clinical infectious diseases : an official publication of the Infectious Diseases Society of America 2008, 46(12):1813-1821.

20. Yu JQ, Tang KJ, Xu BL, Xie CM, Light RW: Pulmonary cryptococcosis in non-AIDS patients. The Brazilian journal of infectious diseases : an official publication of the Brazilian Society of Infectious Diseases 2012, 16(6):531-539.

21. Davda S, Kowa XY, Aziz Z, Ellis S, Cheasty E, Cappocci S, Balan A: The development of pulmonary aspergillosis and its histologic, clinical, and radiologic manifestations. Clinical radiology 2018, 73(11):913-921.

22. Wake RM, Britz E, Sriruttan C, Rukasha I, Omar T, Spencer DC, Nel JS, Mashamaite S, Adelekan A, Chiller TM et al: High Cryptococcal Antigen Titers in Blood Are Predictive of Subclinical Cryptococcal Meningitis Among Human Immunodeficiency Virus-Infected Patients. Clinical infectious diseases : an official publication of the Infectious Diseases Society of America 2018, 66(5):686-692.

23. Chen GL, Chen Y, Zhu CQ, Yang CD, Ye S: Invasive fungal infection in Chinese patients with systemic lupus erythematosus. Clinical rheumatology 2012, 31(7):1087-1091. 
24. Lao M, Zhan Z, Su F, Li H, Yang Z, Chen H, Liang L, Chen D: Invasive mycoses in patients with connective tissue disease from Southern China: clinical features and associated factors. Arthritis research \& therapy 2019, 21(1):71.

25. Kim HJ, Park YJ, Kim WU, Park SH, Cho CS: Invasive fungal infections in patients with systemic lupus erythematosus: experience from affiliated hospitals of Catholic University of Korea. Lupus 2009, 18(7):661-666.

26. Kim JM, Kim KJ, Yoon HS, Kwok SK, Ju JH, Park KS, Cho CS, Kim HY, Park SH: Meningitis in Korean patients with systemic lupus erythematosus: analysis of demographics, clinical features and outcomes; experience from affiliated hospitals of the Catholic University of Korea. Lupus 2011, 20(5):531-536.

27. Lao M, Wang X, Ding M, Yang Z, Chen H, Liang L, Zhan Z, Chen D: Invasive fungal disease in patients with systemic lupus erythematosus from Southern China: a retrospective study. Lupus 2019, 28(1):77-85.

\section{Tables}

Table 1 Basic characteristics of cryptococcosis in patients with CTD 


\begin{tabular}{|c|c|c|c|}
\hline Characteristics & SLE $(n=20)$ & $\operatorname{AAV}(n=6)$ & RA (n=4) \\
\hline \multicolumn{4}{|l|}{ Demographic characteristics } \\
\hline Sex, male:female & $3: 17$ & $4: 2$ & $1: 3$ \\
\hline Age, years, mean \pm SD & $37.5 \pm 12.2$ & $62.5 \pm 13.8$ & $60.3 \pm 2.8$ \\
\hline Duration of CTD, months, median (IQR) & $24.0(6.3,66.0)$ & $36.0(5.0,48.0)$ & $66.0(9.0,150.0)$ \\
\hline Nephritis, n (\%) & $13(65.0)$ & $5(83.3)$ & $0(0)$ \\
\hline \multicolumn{4}{|l|}{ Infective sites } \\
\hline Lung, n (\%) & $8(40.0)$ & $6(100)$ & $4(100)$ \\
\hline Meninges, n (\%) & $6(30.0)$ & $0(0)$ & $0(0)$ \\
\hline Blood stream, n (\%) & $5(25.0)$ & $0(0)$ & $0(0)$ \\
\hline Meninges+Blood stream, n (\%) & $1(5.0)$ & $0(0)$ & $0(0)$ \\
\hline \multicolumn{4}{|l|}{ Laboratory data } \\
\hline WBC count $\left(\times 10^{9} / 1\right)$, mean \pm SD & $7.3 \pm 4.9$ & $6.9 \pm 3.3$ & $5.7 \pm 2.8$ \\
\hline Lymphocyte count $\left(\times 10^{9} / \mathrm{l}\right)$, mean \pm SD & $0.9 \pm 0.5$ & $1.3 \pm 1.1$ & $0.9 \pm 0.7$ \\
\hline Hemoglobin $(\mathrm{g} / \mathrm{l})$, mean $\pm \mathrm{SD}$ & $95.2 \pm 26.4$ & $110.2 \pm 21.1$ & $118.3 \pm 27.9$ \\
\hline Serum albumin $(\mathrm{g} / \mathrm{l})$, mean $\pm \mathrm{SD}$ & $31.8 \pm 6.1$ & $28.7 \pm 8.5$ & $34.8 \pm 5.3$ \\
\hline Elevated serum creatinine, n (\%) & $7(35.0)$ & $4(66.7)$ & $0(0)$ \\
\hline $\operatorname{IgM}(\mathrm{g} / \mathrm{l})$, median (IQR) & $0.6(0.5,0.9)$ & $0.6(0.5,0.8)$ & $1.6(0.9,2.7)$ \\
\hline $\operatorname{IgG}(\mathrm{g} / \mathrm{l})$, median (IQR) & $10.2(8.1,12.2)$ & $11.9(8.6,12.8)$ & $11.9(10.1,12.0)$ \\
\hline ESR $(\mathrm{mm} / \mathrm{h})$, median (IQR) & $24.0(16.0,32.0)$ & $31.0(19.0,34.0)$ & $48.0(25.5,59.0)$ \\
\hline CRP (mg/l), median (IQR) & $31.9(5.2,64.0)$ & $6.2(1.9,73.7)$ & $42.9(7.1,89.4)$ \\
\hline Cryptococcal capsule polyglycan antigen positive, n (\%) & $13(68.4)$ & $5(100)$ & $4(100)$ \\
\hline \multicolumn{4}{|l|}{ Immunosuppresive treatment } \\
\hline Accumulated dose of prednisone, g, median (IQR) & $0.9(0.7,2.4)$ & $1.9(0.3,2.6)$ & $0.5(0,0.9)$ \\
\hline MMF, n (\%) & $7(35.0)$ & $0(0)$ & $0(0)$ \\
\hline CYC, n (\%) & $5(25.0)$ & $1(16.7)$ & $0(0)$ \\
\hline CsA, n (\%) & $2(10.0)$ & $0(0)$ & $0(0)$ \\
\hline MTX, n (\%) & $1(5.0)$ & $0(0)$ & $1(25.0)$ \\
\hline
\end{tabular}

AAV, ANCA-associated vasculitis; CRP, C-reactive protein; CTD, connective tissue disease; CsA, cyclosporin A; CYC, cyclophosphamide; ESR, erythrocyte sedimentation rate; IQR, interquartile range; MMF, mycophenolate mofetil; MTX, methotrexate; RA, rheumatoid arthritis; SD, standard deviation; SLE, systemic lupus erythematosus.

Table 2 Radiographic manifestations of pulmonary cryptococcosis 


\begin{tabular}{ll}
\hline Characteristics & Total (n=18) \\
\hline Radiographic manifestation & \\
\hline Infiltration, $\mathrm{n}(\%)$ & $10(55.6)$ \\
\hline Nodules with diameter <3 cm, n (\%) & $8(44.4)$ \\
\hline Ground glass opacity, n (\%) & $2(11.1)$ \\
\hline Cavitary lesions, n (\%) & $1(5.6)$ \\
\hline Location & \\
\hline Single lobe, n (\%) & $10(55.6)$ \\
\hline Multiple lobes, n (\%) & $8(44.4)$ \\
\hline
\end{tabular}

Table 3 Characteristics of CSF in patients with cryptococcal meningitis

\begin{tabular}{ll}
\hline Characteristics & $\mathbf{N}=7$ \\
\hline Leukocytes $\left(\times 10^{6} / \mathrm{l}\right)$, median (IQR) & $57.0(42.5,66.0)$ \\
\hline Mononuclear cell, \%, mean \pm SD & $78.4 \pm 10.4$ \\
\hline Total protein (mg/l), median (IQR) & $510.0(448.1,699.5)$ \\
\hline Glucose (mmol/l), median (IQR) & $1.8(1.5,2.4)$ \\
\hline Chloride (mmol/l), median (IQR) & $117.0(116.0,119.0)$ \\
\hline Open pressure > 200mm $\mathrm{H}_{2} \mathrm{O}, \mathrm{n}(\%) 6(85.7)$ \\
\hline Indian ink positive, $\mathrm{n}(\%)$ & $6(85.7)$ \\
\hline Serum CrAg positive, $\mathrm{n}(\%)$ & $6(85.7)$ \\
\hline
\end{tabular}

CrAg: cryptococcal antigen; CSF, cerebrospinal fluid; IQR, interquartile range; SD, standard deviation

Table 4 Comparison between CTD patients with and without cryptococcosis 


\begin{tabular}{llll}
\hline Characteristics & Case (n=30) & Control (n=120) & $P$ value \\
& & & \\
\hline Demographic characteristics & & & \\
\hline Sex, male:female & $8: 22$ & 23.97 & 0.36 \\
\hline Age, years, mean+SD & $45.5 \pm 16.3$ & $40.8 \pm 16.6$ & 0.17 \\
\hline Diabetes mellitus, n (\%) & $2(6.7)$ & $5(4.2)$ & 0.56 \\
\hline Duration of CTD, months, median (IQR) & $24.0(6.0,78.0)$ & $24(4.9,84.0)$ & 0.19 \\
\hline Nephritis, n (\%) & $18(60.0)$ & $63(52.5)$ & 0.46 \\
\hline Laboratory data & & & \\
\hline Leukopenia, n (\%) & $7(23.3)$ & $30(25.0)$ & 0.85 \\
\hline Lymphopenia, n (\%) & $18(60.0)$ & $64(53.3)$ & 0.51 \\
\hline Anemia, n (\%) & $18(60.0)$ & $74(61.7)$ & 0.87 \\
\hline Hypoalbuminemia, n (\%) & $20(66.7)$ & $76(63.3)$ & 0.73 \\
\hline Elevated serum creatinine, n (\%) & $11(36.7)$ & $32(26.7)$ & 0.28 \\
\hline IgM (g/l), median (IQR) & $0.7(0.5,1.1)$ & $0.9(0.6,1.4)$ & 0.10 \\
\hline IgG (g/l), median (IQR) & $11.0(8.0,12.3)$ & $13.3(9.4,17.9)$ & $0.001^{*}$ \\
\hline ESR (mm/h),median (IQR) & $28.0(14.0,33.5)$ & $26.5(13.0,55.0)$ & 0.76 \\
\hline CRP (mg/l), median (IQR) & $23.3(5.2,65.4)$ & $2.5(0,14.0)$ & $<0.001^{*}$ \\
\hline Immunosuppressive treatment & & & \\
\hline Accumulated dose of prednisone, g, median (IQR) & $0.9(0.3,2.4)$ & $0.2(0,0.9)$ & $0.01^{*}$ \\
\hline MMF, n (\%) & $7(23.3)$ & $14(11.7)$ & 0.10 \\
\hline CYC, n (\%) & $6(20.0)$ & $20(16.7)$ & 0.67 \\
\hline CsA, n (\%) & $2(6.7)$ & $10(8.3)$ & 0.76 \\
\hline MTX, n (\%) & $2(6.7)$ & $14(11.7)$ & 0.43 \\
\hline
\end{tabular}

CRP, C-reactive protein; CsA, cyclosporin A; CTD, connective tissue disease; CYC, cyclophosphamide; ESR, erythrocyte sedimentation rate; IQR, interquartile range; MMF, mycophenolate mofetil; MTX, methotrexate; SD, standard deviation. $* P<0.05$.

Table 5 Associated factors of cryptococcosis in patients with CTD

\begin{tabular}{|c|c|c|c|c|c|c|}
\hline & \multicolumn{6}{|c|}{ Univariate logistic regression Multivariate logistic regression } \\
\hline Characteristics & Crude OR & $95 \% \mathrm{CI}$ & $P$ value & Adjusted OR S & $95 \% \mathrm{CI}$ & $P$ value \\
\hline Serum IgG, $g / l$ & 0.89 & $0.83-0.97$ & $0.01 *$ & - & - & - \\
\hline Accumulated dose of prednisone, $\mathrm{g}$ & 1.42 & $1.08-1.87$ & $0.01 *$ & 1.42 & 1.08-1.87 & $0.01 *$ \\
\hline
\end{tabular}

CI, confidential interval; CTD: connective tissue disease; OR, odds ratio. ${ }^{*} P<0.05$.

\section{Figures}



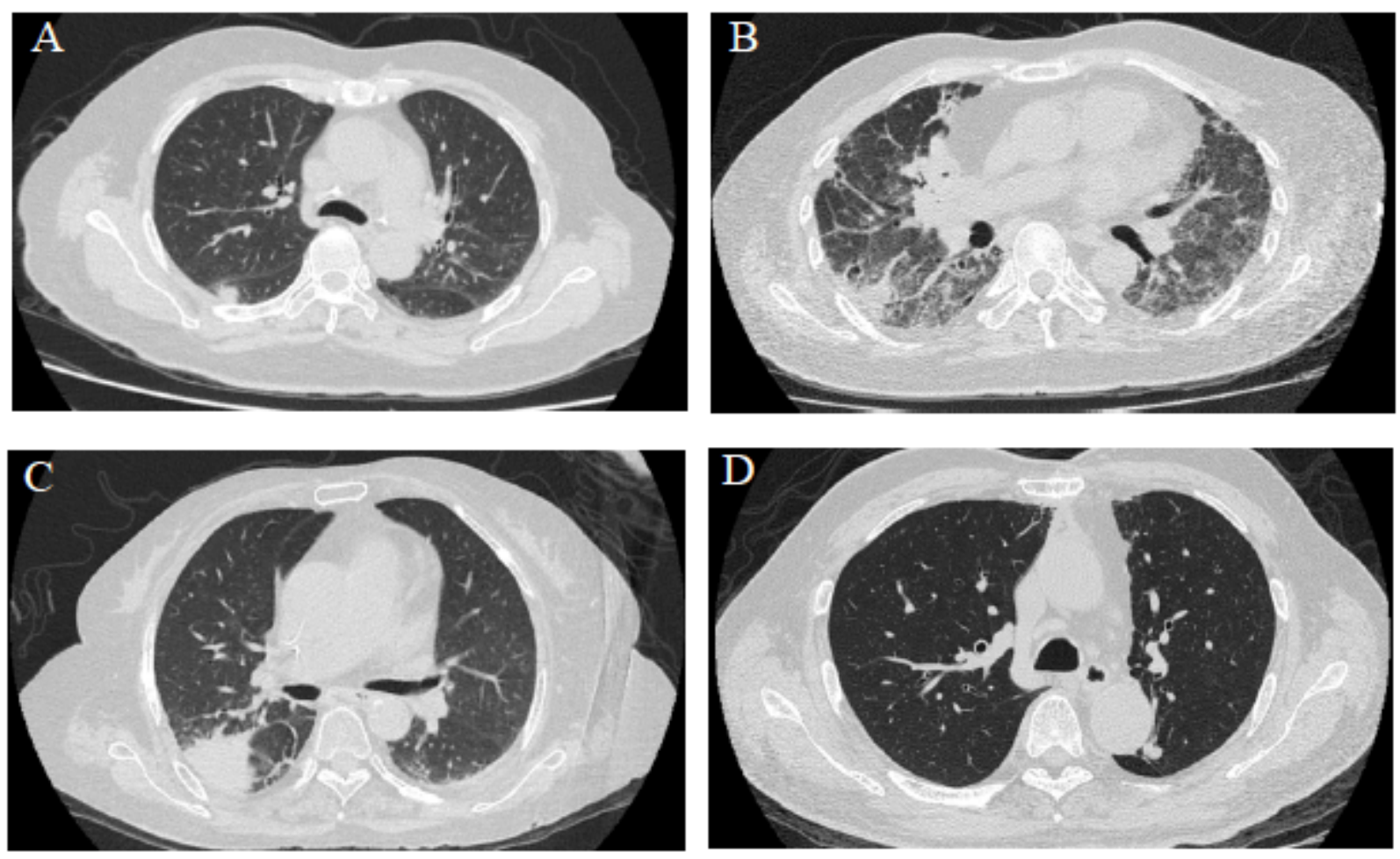

\section{Figure 1}

Manifestations of cryptococcosis in pulmonary computed tomography (CT) scan in patients with connective tissue diseases (CTD). (A) A small nodule with blurred edge in the right lower lung in a patient with ANCA-associated vasculitis (AAV). (B) A new-developed cavitary lesion located in the right lobe in the background of interstitial pneumonia in a patient with AAV. (C) Pulmonary CT scan showed a triangleshaped consolidation in the right upper lobe in a patient with rheumatoid arthritis (RA). (D) A small nodule displayed spiculated margin in left upper lobe in a patient with systemic lupus erythematosus (SLE), which was misdiagnosed with lung cancer.

\section{Supplementary Files}

This is a list of supplementary files associated with this preprint. Click to download.

- supplement1.docx 